\title{
A review of leakage current mechanism in nitride based light emitting diode
}

\author{
Anati Syahirah Hedzir ${ }^{a}$, Norasmahan Muridan ${ }^{b}$, Nurul Fadzlin Hasbullah ${ }^{\mathrm{c}, *}$ \\ ${ }^{a}$ Department of Electrical and Computer Engineering, Kuliyyah of Engineering, International Islamic University Malaysia, 53100 Kuala Lumpur, \\ Malaysia \\ ${ }^{b}$ Department of Electrical and Computer Engineering, Kuliyyah of Engineering, International Islamic University Malaysia, 53100 Kuala Lumpur, \\ Malaysia \\ ${ }^{c}$ Department of Electrical and Computer Engineering, Kuliyyah of Engineering, International Islamic University Malaysia, 53100 Kuala Lumpur, \\ Malaysia \\ * Corresponding author: nfadzlinh@iium.edu.my
}

Article history

Received 10 February 2016

Received in revised form 3 May 2016

Accepted 4 May 2016

\begin{abstract}
We review the dominant mechanism and characteristics which give rise to the existence of forward and reverse leakage current in nitride based light emitting diode (LED). The existence of leakage current can affect the reliability and efficiency of LED. Hence, to understand the mechanism that is responsible for its existence, the occurrence of leakage current is divided into three main parameters: 1) low bias; 2) medium or high bias; 3) temperature dependence. For reverse bias, many reports suggest Poole-Frenkel as the dominant mechanism in low reverse bias and some others suggested the field dependent tunneling mechanism. In high reverse bias, many studies have shown tunneling to be the dominant mechanism. However, there is also few other papers suggesting variable range hopping ( $\mathrm{VRH})$ or $\mathrm{PF}$ being a dominant mechanism. When the reverse bias currentvoltage measurement below $200 \mathrm{~K}$, majority studies reported VRH to be the dominant mechanism. Meanwhile, for forward bias, defect-assisted tunneling is most likely the dominant mechanism. At low forward voltages, electrons are believed to be the dominant carriers for defect assisted tunneling, while holes are reported to be likely the dominant carrier in medium forward voltages.
\end{abstract}

Keywords: Reverse leakage current, forward leakage current, Poole-Frenkel, variable range hopping, tunneling.

(C) 2016 Penerbit UTM Press. All rights reserved

\section{INTRODUCTION}

In recent years, nitride based LED has attracted great interest as one of the promising device that can provide environmental friendly light that has high efficiency [26][27]. In addition, nitride based LEDs are also considered as an excellent device due to its fast modulation speed, good robustness to shocks and atmospheric agents and for having linear behavior under continuous current reduction and pulsewidth modulation dimming. Furthermore, it is also considering as one of the core component that is expected to replace the incandescent and compact fluorescent lamps (CFLs), indicating the start of the new era of solid state lighting [24]. LED-based illuminating devices are free from mercury unlike CFLs and this makes LED much safer compared CFLs that can be consider edas a hazardous material.

In addition to its luminescence efficiency, the existence of leakage current in this devices should be giving enough attention as well. The existence of leakage current indicates the extent of the device quality, reliability, and electrostatic discharge [1][2][25]. Cao et al. [41] and Garam et. al. [31] stated that the degradation of optical power is often linked to the existence of leakage current. Hence, the aim of this paper is to review the dominant mechanism responsible for the occurrence of leakage current in forward and reverse bias region. LEDs with reduced leakage current are most desirable in space and communication industry. Hence it is important to understand the underlying mechanism behind leakage current to improve the technology of LEDs [8].

In the second section, we will review the dominant mechanism that is responsible for the presence of leakage current in low and high reverse bias. On the next section, the dominant mechanism responsible for forward leakage current in low and medium bias will be discussed. The last section will be the conclusion.

\section{REVERSE BIAS REGION}

When there is an externally applied potential in the junction, the Fermi level will no longer remain in equilibrium throughout the system. In reverse biased, the electron which is located in the n-type and holes that stay on the p-type are being swept away by the electric field inside the space-charged region. Supposedly, there should be no flow of current, however, small current still present due to the diffusion of minority carriers to majority carriers and it is known as leakage current. The mechanism that is responsible for the presence of leakage current will be discussed in details in the next section. 
Table 1 Summary of the dominant leakage current mechanism in low reverse bias region

\begin{tabular}{|c|c|c|c|c|c|}
\hline Refs. & Year & Mechanism & Characteristic & Strength & Limitation \\
\hline [12] & 1999 & PF & $\begin{array}{l}\text { Low electric field } \\
(<1 \mathrm{Kv} / \mathrm{cm})\end{array}$ & $\begin{array}{l}\text { - Explain the equation used to } \\
\text { fit with the measured data } \\
\text { - Explain method to distinguish } \\
\text { PF and PAT }\end{array}$ & $\begin{array}{l}\text { - Does not state specific temperature of } \\
\text { conduction for both mechanism }\end{array}$ \\
\hline [13] & 2002 & $\begin{array}{l}\text { Field } \\
\text { dependent } \\
\text { tunneling }\end{array}$ & 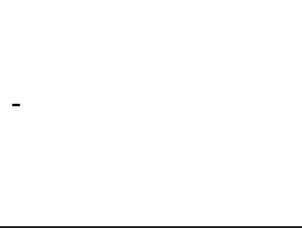 & $\begin{array}{l}\text {-Explain the specific region of } \\
\text { bias for the dominance of each } \\
\text { tunneling mechanism }\end{array}$ & $\begin{array}{l}\text {-Does not state specific range of applied } \\
\text { voltage and temperature to indicates the start } \\
\text { and end of the respective mechanism } \\
\text {-Does not use fitting technique to support the } \\
\text { suggested mechanism }\end{array}$ \\
\hline [11] & 2011 & $\mathrm{PF}$ & $\begin{array}{l}\text {-Low electric field }(< \\
\left.2.5 \times 10^{6} \mathrm{~V} / \mathrm{cm}\right) \\
\text {-High temperature } \\
(260 \mathrm{~K}-400 \mathrm{~K})\end{array}$ & $\begin{array}{l}\text {-Specify the range of electric } \\
\text { field that indicates the } \\
\text { dominance of each mechanism } \\
\text { - Specify the temperature of } \\
\text { conduction for PF and PAT }\end{array}$ & $\begin{array}{l}\text { - The theory of DT is not proven in this paper } \\
\text { because the measured data is not being fitted } \\
\text { with any theoretical equation }\end{array}$ \\
\hline$[10]$ & 2012 & PF & $\begin{array}{l}\text {-Dominant up to } \sim \text { - } \\
17.9 \mathrm{~V} \\
\text {-High temperature } \\
\text { (above } 280 \mathrm{~K} \text { ) }\end{array}$ & $\begin{array}{l}\text {-specify the temperature of } \\
\text { conduction for each mechanism } \\
\text { to takes place } \\
\text {-List down the equation used for } \\
\text { fitting purposes }\end{array}$ & $\begin{array}{l}\text { - Does not specify the range of voltage that } \\
\text { indicates the dominance of each mechanism }\end{array}$ \\
\hline [9] & 2015 & $\mathrm{PF}$ & $\begin{array}{l}\text {-From }-2 \mathrm{~V} \text { up to }-10 \\
\mathrm{~V} \\
\text {-High temperature } \\
(300 \mathrm{~K}-400 \mathrm{~K})\end{array}$ & $\begin{array}{l}\text {-Specify the range of applied } \\
\text { voltage and temperature that } \\
\text { indicates the dominance of each } \\
\text { mechanism } \\
\text {-Fitting each measured data } \\
\text { with theoretical equation }\end{array}$ & $\begin{array}{l}\text {-Does not specify in details the relation } \\
\text { between the existence of threading location } \\
\text { that can lead to the existence of VRH and PF. }\end{array}$ \\
\hline
\end{tabular}

\section{Low reverse bias region}

In recent years, there have been several papers reporting on the reverse leakage current characteristics of nitride based LED [1]-[8]. In this section, we will investigate the mechanism responsible for the formation of leakage current in low reverse bias and its characteristics.

Among the method widely used to study the mechanism behind leakage current is by investigating the relation between leakage current versus electric field and the leakage current versus temperature. This method can be seen in a report conducted by Han $e$. al. [9] where two different mechanisms were determined through this method. When the range of applied voltage from $-2 \mathrm{~V}$ up to $-10 \mathrm{~V}$, report by [9] claims that the Poole Frenkel (PF) is the dominant mechanism and from $-10 \mathrm{~V}$ onwards, $\mathrm{PF}$ is outweighed by variable range hopping (VRH) mechanism. PF mechanism is expected to be the dominant electrical conduction mechanism when the depletion region of the semiconductor contains large trap density and are thick enough to avoid quantum mechanical tunneling.

Due to the presence of traps, the movement of electrons through drift and diffusion process from the conduction band reduce. Hence, causing the trapping and de-trapping of electrons become the dominant process. Occasionally, random thermal fluctuation will give the trapped electron enough energy to excite from the trap states to the conduction band, inducing leakage current. As the reverse voltage increases, the depletion slope becomes steeper and thus causing the lowering of PF barrier. Due to this, it will be easier for the excitation process of trapped electrons into the conduction band to occur. Hence, the reverse leakage current increase proportional with the amount of trap and applied bias voltage [29]. To support this theory, the measured data can be fitted with eq. (1). If eq. (1) gives out the value of $\sim 0.5, \mathrm{PF}$ is the dominant mechanism and if eq. (1) gives out the value of $\sim 1, \mathrm{VRH}$ is the dominant mechanism [9].

$$
d[\log (\ln I)] / d(\log E)]
$$

Furthermore, it is believed that PF dominates the current conduction mechanism when the temperature is above $200 \mathrm{~K}$ [9]-[12][28]. A report by [10] stated that the dominance of PF can also be proved by fitting the following equation with the measured data:

$$
\begin{aligned}
& I \propto \exp \left\{-\frac{E_{a}}{k T}\right\} \\
& E_{a}=\phi_{P F}-\beta_{P F} F^{\frac{1}{2}}
\end{aligned}
$$

where $E_{a}$ is the thermal activation energy, $\mathrm{k}$ is the Boltzmann constant, $\phi_{P F}$ is the barrier height of carriers trapped in deep centers without external electric field, $\beta_{P F}$ is the Poole-Frenkel constant and $F$ is the local electric field strength exerting on the deep centers. In theory, $\epsilon_{P F}=\left(\frac{q}{\pi \epsilon}\right)^{\frac{1}{2}}$ where $\mathrm{q}$ is the electric charge and $\epsilon$ is the permittivity of material at high frequency. This expression calculates the theoretical value of the Poole-Frenkel constant which yields a value of $3.28 \times 10^{-4} \mathrm{eV} \cdot \mathrm{v}^{\frac{-1}{2}} . \mathrm{cm}^{\frac{1}{2}}$. By linear fitting eq. (2) with the plot of thermal activation energy from an Arrhenius plot against the square root of reverse bias, it can be proved that the range of temperature for the conduction PF mechanism is from $280-400 \mathrm{~K}$. Moreover, through fitting eq. (2) with measured I-V, it can be seen that PF mechanism is dominant up to $\sim-17.9 \mathrm{~V}$ and not only limited up to $-10 \mathrm{~V}$.

Meanwhile, a report by [11] and [12] characterize the dominant mechanism through the range of applied electric field instead of applied voltage and temperature. The study conducted by 
[11] conclude that at low electric field range $\left(<2.5 \times 10^{6} \mathrm{~V} / \mathrm{cm}\right)$, the lowering of PF barrier occurs and hence it become the path for the leakage current. The lowering of the barrier stated in [11] explains through equation shown below:

$$
\begin{aligned}
& I_{P F} \propto F \exp \left(-\frac{W}{k T}\right) \\
& W=\Phi_{P F}-\beta_{P F} F^{1 / 2} \\
& \beta_{P F}=\left(q / \pi \varepsilon_{0} \varepsilon_{r}\right)^{1 / 2}
\end{aligned}
$$

where $\varepsilon_{0}$ is the permittivity of space, $\varepsilon_{r}$ is the permittivity of the material, $\Phi_{P F}$ is the PF barrier height in the absence of a biased electric field, and $F$ is the local electric field. Meanwhile, report by Ganichev et al. [12] stated that the sufficient low-electric field strengths for the dominance of $\mathrm{PF}$ are below $1 \mathrm{Kv} / \mathrm{cm}$. In this paper, the theory is extracted by studying the relation between emission rate and electric field. The dependence of emission rate on electric field can be express as $(\ln (\mathrm{e}) \propto \sqrt{E})$, where the plot of $\ln (\mathrm{e})$ against $\sqrt{E}$ is fitted with a straight line. While many papers suggest $\mathrm{PF}$ as the dominant mechanism for low bias condition, however, there is a probability of dominance from other type of mechanism.

A report by [13] attribute the existence of reverse current in low bias due to field-dependent tunneling instead of PF. The discussion in this paper suggests that the existence of the defect in the space charge region promotes the process of field-dependent tunneling which ultimately contribute to the existence of leakage current. Table 1 summarizes of all afore mentioned reports on the probable leakage current mechanism in low reverse bias region. From this table, it can be understood that:

1) $\mathrm{PF}$ is relatively dominant at low bias.

2) PF conduction is at low electric field.

3) $\mathrm{PF}$ conduction is at high temperature (above $200 \mathrm{~K}$ ).

\section{High reverse bias region}

Due to high reverse voltage the mechanism that is responsible for the leakage current maybe different from the previous section. As the range of electric field present in the space charge region become higher i.e. $\quad<8 \times 10^{7} \mathrm{~V} / \mathrm{cm}$, phonon-assisted tunneling (PAT) is believed has become the main mechanism for the existence of leakage current in high bias [11]. Phonon assisted tunneling is described as the process of a trapped carrier in the deep levels tunneling through the space charge region barrier with the aid of phonon emission. Report in [11] described the emission current by PAT as below:

$$
I_{P A T} \propto \sum_{\rho} \pi_{\rho} \Gamma\left(\Delta_{\rho}\right)
$$

With,

$$
\begin{aligned}
& \Gamma(\Delta)=\gamma \frac{\Delta}{q K} e^{-K}, \\
& K=\frac{4 \sqrt{2 m^{*}} \Delta^{\frac{3}{2}}}{3 \hbar F} \\
& \Pi_{\rho}=\left(1-e^{-\frac{\hbar w}{k T}}\right) \sum_{n=0}^{\infty} e^{-\frac{\hbar w}{k T} J_{\rho}^{2}} \\
& \mathrm{X}\left[2 \sqrt{S\left(n+\frac{1}{2}\right)}\right]
\end{aligned}
$$

where $\Pi_{\rho}$ represents the occupation probability of quasi level with phonon energy $\rho \hbar \omega$ (here $\rho$ is an integer) while $\Gamma\left(\Delta_{\rho}\right)$ represents the tunneling ionization rate at the quasi level, $\gamma$ is the fitting parameter, $\Delta$ is the barrier height, $m^{*}$ is the effective mass of electron, $J_{\rho}$ is the Bessel function of the first kind, $T$ is the temperature, $S$ is the HuangRhys factor. By fitting the calculated leakage current with measured data, PAT proved to be dominant on high range of applied electric field as well as in high temperature situation $\left(70^{\circ} \mathrm{C}\right.$ and higher). Similar findings have also been reported in [12], where it is believed that by increasing the electric field beyond $1 \mathrm{Kv} / \mathrm{cm}$, the field dependence of the emission rate will experience a change from $\ln (e) \propto \sqrt{E}$ to $\ln (e) \propto E^{2}$, which indicating a change from PF to PAT respectively. To determine the dominance between these two mechanisms, the logarithmic plot of emission rate is fitted onto $\sqrt{E}$ and $E^{2}$. A Plot that is well fitted by a straight line will indicate the dominant mechanism. Despite giving out a different range of applied electric field that marks the dominance of PAT, [11] and [12] agreed that PAT is the dominant mechanism in high bias region. However, there is a limitation to how far the dominance of PAT extends in high bias region. According to a report in [12], the electric field range of PAT dominance is limited to field strengths of

$$
E \leq E^{*}=\sqrt{2 m^{*} E_{T}} /\left(e \tau_{2}\right)
$$

When the electric field exceeds this range, it is believed that direct tunneling (DT) is the dominant mechanism responsible for the carrier emission process. However, this theory is not proven through fitting a measured data with calculated data because DT requires much higher electric field than this paper intended to discuss. The theory of DT is later supported in [11], given with a clear range of electric field that indicates its dominance. In the paper, it is stated that when the electric field present in the space charge region become too strong e.g. electric field $>8 \times 10^{7} \mathrm{~V} / \mathrm{cm}$, PAT will be outweighed by DT. However, there is no measured data provided in this paper to support the theory which suggest, the dominance of DT is still unclear.

Aside from PAT mechanism, there are other reports that suggest different theories. A paper by Cao et al. [13] claims that band-to-band tunneling is dominant high reverse bias because the data that has been obtained is in good agreement with band-to-band tunneling. It is believed that the presence of defects located in cladding layer of the devices enhanced the dominance of band-to-band tunneling, resulting in an increase of reverse leakage current [41]. However, they did not fit the measured data with any calculation. Hence, the range of applied voltage that indicates the start and end of band-to-band tunneling dominance is not clear. Furthermore, we observed that there is also a possibility of VRH being the dominant mechanism [9]. In this paper, the range of dominance for VRH mechanism is defined from $-11 \mathrm{~V}$ up to $-20 \mathrm{~V}$. Based on this paper, when the slope of eq. (1) is equal to $\sim 1$, the dominant mechanism is VRH.

Meanwhile, report by [29] and [30] believed that PF is the dominant mechanism responsible for the existence of leakage current in high reverse bias. By fitting eq. (11) with measured data, the graph is best fit when the voltage range is from $-11 \mathrm{~V}$ up to $-18 \mathrm{~V}$ [29]. Furthermore, based on temperature dependence measurement, it has proven that within the temperature range of $295 \mathrm{~K}-520 \mathrm{~K}$, PF is the dominant mechanism in high reverse bias and leakage current was found to increase proportionally with reverse voltage and temperature [29].

$$
I=I_{o} \exp \left(\frac{\beta_{P F} F^{\frac{1}{2}}}{k T}\right)
$$

By reviewing papers related to high reverse bias, we observed that different paper came out with different theory and characterization. Table 2. summarizes the mechanism that is responsible for leakage current in high reverse bias. 
Table 2 Dominant mechanism for reverse leakage current in high bias

\begin{tabular}{|c|c|c|c|c|c|}
\hline Refs & Year & Mechanism & Characteristics & Strength & Limitation \\
\hline [12] & 1999 & PAT & $\begin{array}{l}\text { High electric field (> } \\
1 \mathrm{Kv} / \mathrm{cm})\end{array}$ & $\begin{array}{l}\text { - Explain the equation used to fit } \\
\text { with the measured data }\end{array}$ & $\begin{array}{l}\text { - Does not state specific temperature of } \\
\text { conduction for both mechanism }\end{array}$ \\
\hline [13] & 2002 & $\begin{array}{l}\text { Band -to- band } \\
\text { tunneling }\end{array}$ & - & $\begin{array}{l}\text {-Explain the specific region of } \\
\text { bias for the dominance of each } \\
\text { tunneling mechanism }\end{array}$ & $\begin{array}{l}\text {-Does not state specific range of applied } \\
\text { voltage and temperature to indicates the } \\
\text { start and end of the respective mechanism }\end{array}$ \\
\hline [29] & 2007 & $\mathrm{PF}$ & $-11 \mathrm{~V}$ up to $-18 \mathrm{~V}$ & $\begin{array}{l}\text {-Specify the range of electric field } \\
\text {-Fitting each measured data with } \\
\text { theoretical equation }\end{array}$ & $\begin{array}{l}\text { - Does not specify the range of electric } \\
\text { field that leads to the existence of PF. }\end{array}$ \\
\hline \multirow[t]{2}{*}{ [11] } & \multirow[t]{2}{*}{2011} & PAT & $\begin{array}{l}\text { High electric field }(< \\
\left.8 \times 10^{7} \mathrm{~V} / \mathrm{cm}\right)\end{array}$ & \multirow{2}{*}{$\begin{array}{l}\text {-Specify the range of electric field } \\
\text { - Fitting each measured data with } \\
\text { theoretical equation }\end{array}$} & \multirow[t]{2}{*}{$\begin{array}{l}\text {-The theory of DT is not proven in this } \\
\text { paper because the measured data is not } \\
\text { being fitted with any theoretical equation }\end{array}$} \\
\hline & & DT & $\begin{array}{l}\text { High electric field (> } \\
\left.8 \times 10^{7} \mathrm{~V} / \mathrm{cm}\right)\end{array}$ & & \\
\hline [9] & 2015 & VRH & $-11 \mathrm{~V}$ up to $-20 \mathrm{~V}$ & $\begin{array}{l}\text {-The range of applied voltage is } \\
\text { specified } \\
\text {-Fitting each measured data with } \\
\text { theoretical equation }\end{array}$ & $\begin{array}{l}\text {-Does not specify in details the relation } \\
\text { between the existence of threading } \\
\text { location that can leads to the existence of } \\
\text { VRH. }\end{array}$ \\
\hline [30] & 2015 & $\mathrm{PF}$ & - & $\begin{array}{l}\text {-Explain the specific region of } \\
\text { bias for the dominance of each } \\
\text { mechanism. }\end{array}$ & $\begin{array}{l}\text {-Does not state specific range of applied } \\
\text { voltage and temperature to indicates the } \\
\text { start and end of the respective mechanism } \\
\text { - Does not fit any measured data with } \\
\text { theoretical equation }\end{array}$ \\
\hline
\end{tabular}

\section{Temperature Dependence}

In the previous section, the parameters that define the dominant mechanism in reverse leakage current were divided into region of bias. However, there are several papers that discuss about the dominance of specific mechanism with no clear state of the bias region of the mechanism. They identify the mechanism through different parameters, such as temperature dependence. Report by [14] believed that when the temperature reached $250 \mathrm{~K}$ and lower, the reverse leakage current is mainly attributed to the VRH conduction and this assumption is made based on Fig.1.

Based on the result of the measurement in Fig.4, it is observed that, at low temperature, there is enough thermal energy for the carrier to hop further a far but still within the accessible energy band around the Fermi level. When the activation energy of the nearest trap is large, a hop to a further trap with lower activation energy is much easier. This type of mechanism is known as variable range hopping. The hopping distance of the electron depends on the temperature.

According to this paper, the main hopping carriers at low temperature in a semi-insulating $\mathrm{GaN}$ is an electron. The electron will hop from one trap to another trap. When the pn-junction is in reverse bias, the space charge region is considering as an insulating layer with a strong electric field. Under this condition, the equation of current can be expressed as follow:

$$
I=I_{o} \exp \left[-C\left(T_{O} / T\right)^{1 / 4}\left(1-C^{\prime} F^{2} / T^{1 / 2}\right)\right]
$$

where $C$ and $C^{\prime}$ are constant and $F$ is the electric field. In a limited range of temperature, the temperature dependence of the leakage current is small (from $80 \mathrm{~K}$ to $250 \mathrm{~K}$ ). Hence, the reverse current obeys the Mott's $T^{-1 / 4}$ law when the value of voltage is fixed. Mott's theory stated that the conductivity of VRH in a disordered material can be expressed through $\sigma(T) \propto \exp \left[-\left(T_{O} / T\right)^{1 / 4}\right]$, where $T_{O}$ is the characteristic temperature. By fitting eq. (11) with the measured data, the dominance of VRH can be seen as in Fig.1.

\section{Temperature $T(\mathrm{~K})$}

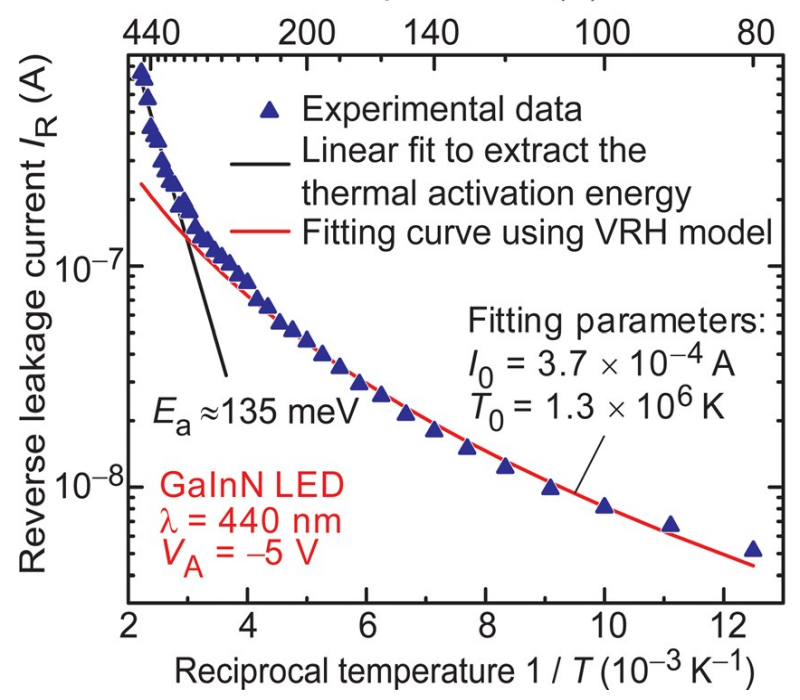

Fig.1 Arrhenius plot of reverse leakage current versus $1 / T$. A VRH conduction mechanism is being used to fit the measured data within the range of $80 \mathrm{~K}-440 \mathrm{~K}$. Copyright 2011 Shan et. al. 
Similar findings have also been reported in [9] and [16]. Initially, report by [10] stated in his earlier paper that when the temperature of conduction reaches below $280 \mathrm{~K}$, the mechanism associated to the reverse leakage current is VRH. The relation between current and temperature can be explained as follow:

$$
I \propto I_{0} \exp \left\{-{\frac{T_{0}}{T}}^{1 / 4}\right\}
$$

where $T_{0}$ is the characteristic temperature. By fitting eq. (12) onto the measured data, we can determine the possibility for VRH in agreement with the assumption. However, in his recent paper, he claimed that the range of temperature should be lower than $280 \mathrm{~K}$ [8], which in this case is $<200 \mathrm{~K}$ in order for the VRH mechanism to take place and this presumption is supported by a series of Arrhenius plot with range of temperature from $80 \mathrm{~K}-400 \mathrm{~K}$ [16]. As reported in [9], [14], [16] and [28], all claimed that VRH is the main mechanism responsible for reverse leakage current at low temperature (below 200 $\mathrm{K})$. It is believed that the dominance of VRH is due to the presence of threading dislocation in the device [15][32] - [36].

However, there are other researchers who argue that VRH may not necessarily be the dominant mechanism in reverse bias at low temperature. Miller et al. [15] believe that field-emission tunneling is the mechanism that is dominant at low temperature. The emission of electrons from the semiconductor into dielectric under the influence of an electric field is known as field emission tunneling. In this mechanism, electrons that tunnel through a potential barrier will exponentially decay. Hence, there is a finite probability that the electron will be found outside of the barrier. The current flow due to tunneling of electrons is given by:

$$
J_{m \rightarrow s}=\left(A^{*} \mathrm{~T}\right) / \mathrm{k} \int_{0}^{q V_{b}} F_{m} T(\eta)\left(1-F_{S}\right) d_{\eta}
$$

where $\mathrm{A}^{*}$ is the effective Richardson's constant, $\mathrm{T}$ is the absolute temperature, $\mathrm{k}$ is Boltzmann's constant, $F_{m}$ and $F_{S}$ are the FermiDirac distribution functions in the metal and semiconductor, $q V_{b}$ is the difference in energy between the top of the Schottky barrier and the conduction band of the undepleted semiconductor, $\eta$ is the energy below the top of the Schottky barrier, and $\mathrm{T}(\eta)$ is the quantum transmission coefficient of the electrons through an approximate triangular Schottky barrier, given by:

$$
T(\eta)=\exp \left(-\frac{\eta^{\frac{3}{2}}}{\sqrt{q V_{b}}} \frac{8}{3} \sqrt{\frac{m * \epsilon_{s}}{N_{D}}} \frac{1}{q h}\right)
$$

where $m *$ is the semiconductor electron effective mass, $N_{D}$ is the donor concentration, $q$ is the electron charge, $\epsilon_{s}$ is the semiconductor dielectric constant, and $h$ is the reduced Planck's constant. Under reverse bias, $\left(1-F_{S}\right) \sim 1$ because the conduction band is considering as unoccupied.The parameters used to fit the tunneling-model based on Miller et. al. reports with the measured data are $A^{*}, \phi_{b}$, and the ratio of $N_{D}$ to the relative effective mass, $\beta=N_{D} /\left(\frac{m^{*}}{m_{o}}\right)$. By fitting these parameters with the measured data, it was found that the range of temperature for VRH to take place is approximately from $125 \mathrm{~K}$ up to $250 \mathrm{~K}$.

\section{FORWARD BIAS REGION}

In the previous section, we reviewed the dominant mechanism that is responsible for the occurrence of leakage current in reverse bias region. It can be seen that the dominance of each mechanism can be divided into different parameters. This section on the other hand, we will review the mechanism associated with leakage current in the forward bias region.

In forward bias, the positive terminal of the voltage source is connected to the p-type semiconductor while the negative terminal is connected to the n-type semiconductor. Due to this condition, the holes in the P-type region and the electrons in the N-type region will relatively diffuse to the opposite region. As a result, the size of the depletion region width reduces. Moreover, when the width of the space region decreasing, the potential barrier in the junction will proportionally decrease. Eventually, the space charge region will become thinner as the forward bias voltage increases. Because of this situation, there is a flow of diffusion current in the pn-junction. However, there is still a presence of small leakage current in a certain region of forward bias. This will be review further in the next section.

\section{Low Forward Bias Region.}

Continuous usage of LED is one of the causes that lead to the increasing amount of leakage current in the forward region. In general, under low forward bias, the defect-assisted tunneling (DAT) is mainly the dominant mechanism responsible for the occurrence of leakage current by [18]- [20][43]. The dominance of defect assisted tunneling is often associated with an unrealistically high ideality factor [4][48][49]. In Fig.2, high ideality factor is seen presents throughout low forward bias region range from $1.2 \mathrm{~V} \sim 1.8 \mathrm{~V}$. The presence of high ideality factor is mainly due to the high dislocation density of the device. To determine the value of the ideality factor, the measured forward I-V data are fitted by the diode characteristics equation shown below:

$$
I=I_{0} \exp \left(\frac{q V}{E_{T}}\right)
$$

where $I_{0}$ is the pre-exponential factor, $q$ is the elementary charge, $V$ is the bias applied to LED device, and $E_{T}$ is the characteristic energy that represents the transparency of tunneling in the space charge region. $E_{T}$ gives the ideality factor of a diode, $n$ through $E_{T}=n k T$. By fitting eq. (14) with the measured forward I-V, the graph of $E_{T} \mathrm{vs}$ temperature can be plotted as in Fig.2. From the graph, it can be seen that the value of ideality factor is unrealistically high thus, indicates the presence of defect assisted tunneling and the dominance of recombination current. However, there are several studies that believed high ideality factor indicates the dominance of trap assisted tunneling (TAT) [43]-[46][13][50]. Hence to distinguished between the dominance of DAT and TAT, the behavior of $E_{T}$, temperature, and ideality factor were plot on the same graph as shown in Fig.6. It can be seen that the behavior of $E_{T}$ is almost constant and it is not dependent on temperature. This is commonly considered as one of the signature of defect assisted tunneling dominance [18][37] - [39].

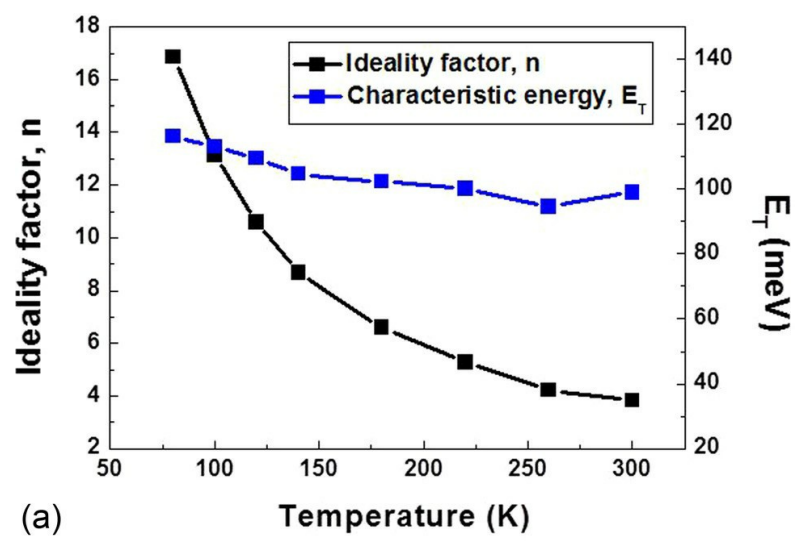

Fig.2 The extracted characteristic energies $E_{T}$ and ideality factor $n$ in low bias at the range of $1.2 \mathrm{~V}<\mathrm{V}<1.8 \mathrm{~V}$. Copyright 2013 Jaekyung et. al.

Even though defect assisted tunneling has been identified as the dominant mechanism in forward bias, there is much info needed to be discussed on the carriers associated with the tunneling. The role of carriers depends on the region of bias. In this case, electron is suggested to be the dominant carriers in defect assisted tunneling. Furthermore, [18] and [19] suggest in low forward bias, electron carriers are dominant in tunneling mechanism and this is proven by comparing the ratio of $E_{T}$ in low and medium bias region into equation below: 


$$
E_{T} \approx \frac{4 e h}{\pi} \sqrt{\frac{N_{I}}{m^{*} \varepsilon_{0} \varepsilon_{r}}}
$$

where $N_{I}$ is the reduced doping level at the space charge region edge, and $m^{*}$ is the effective mass of the specific type of carriers for deep level tunneling. $N_{I}$ can be approximated as the apparent doping concentration $\left(\sim 2.5 \times 10^{17} \mathrm{~cm}\right)$ which can be determined from the capacitance-voltage measurement. In small forward bias, $E_{T}$ can be used to described the barrier of multi-step tunneling between defect sites. Through this, the dominant tunneling carriers can be identified. By comparing the ratio of $E_{T 1}$ (low bias) and $E_{T 2}$ (medium bias) with eq. (15), we will get $E_{T 1} / E_{T 2}=\left(m_{2}^{*} / m_{1}^{*}\right)^{1 / 2}$, that associates the ratio of characteristic energy with the effective mass of the tunneling entities. In $\mathrm{GaN}$, the value of $m^{*}$ for electrons, light holes, and heavy holes are $0.2 m_{0}, 0.3 m_{0}$, and $1.4 m_{0}$ respectively. By substituting the experimental value of $E_{T 1}$ and $E_{T 2}$ into $E_{T 1} / E_{T 2}$, it gives out the value of $\sim 2.4$, which is consistent with the ratio of the square root of $m^{*}$ for heavy holes and electrons $(\sim 2.65)$.

From this result, a report by [19] concluded that electron entities are the dominant entities in defect assisted tunneling mechanism, however, the author does not specify the range of bias that marks the dominance of electron entities. The range of dominance for electron tunneling carriers given by [18] are $1.2 \mathrm{~V} \sim 1.8 \mathrm{~V}$ while range given by [21] and [40] are from $0.3 \mathrm{~V} \sim 1.5 \mathrm{~V}$ and $1.5 \mathrm{~V} \sim 2.1 \mathrm{~V}$. Despite having different range of applied voltage, [18], [19], [21] and [40] agreed that electrons are the dominant tunneling carriers in low bias region

Meanwhile, report by [20] also agreed that defect assisted tunneling is the dominant mechanism for the existence of leakage current in low bias region, however, the author does not specify the respective type of tunneling carriers. In [20], the measured data are fitted with following Mott's law equation:

$$
I(T) \sim \exp \left[-\left(\frac{T_{o}}{T}\right)^{\frac{1}{4}}\right]
$$

where $T_{o}$ is the characteristic temperature. The measured data can be well fitted with Mott's Law equation and this dependence is often associated as the typical features of defect assisted tunneling in a pnjunction diode. The existence of these defect that results in leakage is associated with the present of threading dislocations and V-defects.

However, reports by [22] and [42] believed that trap assisted tunneling (TAT) is the dominant mechanism while report by [47] associates open-core screw dislocations as the main reason for the occurrence of leakage current. In [22], the approach used to prove the assumption is by fitting the parameters of numerical simulated I-V characteristics and a model of trap assisted tunneling to the measured characteristics of different devices. Meanwhile in [42], the theory is proved through a good fit between measured data and simulated forward characteristics of nitride based LED using the basic formulation of Hurkx and Schenk TAT model.

\section{Medium Forward Bias Region.}

Several studies have shown that the existence of leakage current in the forward bias is not confined only in the low bias, instead, it is capable of existing up to medium bias. Reports by [18] [19] [21] and [43] stated that the defect assisted tunneling is the dominant mechanism that is responsible for the existence of leakage current in both low and medium forward bias, however, the difference between those regions are the type of dominant tunneling carriers. For the existence of leakage current in medium bias, hole carriers are more dominant compared to the electron carriers. This can be proved by comparing the ratio of $E_{T}$ in low and medium bias region into eq. (15). Through this, it can be proved that the range of dominance for hole entities is in between $1.6 \mathrm{~V} \sim 2.5 \mathrm{~V}$ [18] [19] [43]. However, a report by [21] use different equation to prove his theory which in this case, the tunneling current $I$, is expressed as:

$$
I=B \exp (\alpha V)
$$

where $\mathrm{B}$ is a constant and $\mathrm{V}$ is the applied forward bias. Meanwhile $\alpha$ in eq. (17) is given by:

$$
\alpha=\pi m_{T}^{1 / 2} \varepsilon^{1 / 2} / 4 h N_{D}^{1 / 2}
$$

where $m_{T}$ is the effective mass of the tunneling entity, $\varepsilon$ is the relative permittivity, $h$ is Plank's constant, and $N_{D}$ is the ionized donor concentration. By analyzing the slopes $(\alpha)$ of $\log I$ vs $V$ plot in different forward bias region, the dominant tunneling carriers can be determined. When other parameters in eq. (18) are held constant, the ratio of the slopes in low and medium bias region are compared with eq. (18) and it is reported that the slopes were consistent with the ratio of the square root of the effective masses of the tunneling carriers. From this method of calculation, Reynolds et. al. [21] stated that the range of applied bias that indicates the dominance of hole carriers for InGaN LED grown on sapphire and GaN substrate are $1.9 \mathrm{~V}-2.4 \mathrm{~V}$.

Based on papers that have been reviewed, it can be understood that majority of the researchers agreed on the same theory despite the difference in approach analysis. It can be concluded that the defect assisted tunneling is the dominant mechanism that leads to the presence of leakage current in both low and medium regions of the forward bias. However, when the amount of applied voltage exceeds the range of hole entities, the value of ideality factor will decrease and become close to two or below. This suggests the transition from tunneling to diffusion current.

\section{CONCLUSION}

In this paper, we have presented a detailed analysis on the dominant mechanism that is responsible for the occurrence of leakage current in reverse and forward bias. The analysis was aim at identifying the type of mechanism that is dominant under specific parameters and the characteristics of each mechanism. In general, we divided our analysis into the following: 1) the dominant mechanism in low reverse and forward bias region; 2) the dominant mechanism in high reverse bias region; 3) the dominant mechanism in medium forward bias region and 4) mechanism subjected to temperature dependence. From this we have found the following:

1) In low reverse bias region, $P F$ is most likely the dominant mechanism. PF need relatively low applied voltage and electric field for it to become dominant. Furthermore, it also dominant in high temperature conduction (above $260 \mathrm{~K}$ up to $400 \mathrm{~K}$ ).

2) In high reverse bias region, tunneling mechanism is most likely the dominant mechanism. However, there are different types of tunneling can be associated with the leakage current. Phonon Assisted Tunneling is reported to be dominant when the range of applied electric field is more than $1 \mathrm{Kv} / \mathrm{cm}$ or $<8 \times 10^{7} \mathrm{~V} / \mathrm{cm}$. While direct tunneling is dominant when the applied electric field exceeds $8 \mathrm{X}$ $10^{7} \mathrm{~V} / \mathrm{cm}$. Nevertheless, VRH is reported to be dominant when the range of applied voltage is from $-11 \mathrm{~V}$ up to $-20 \mathrm{~V}$.

3) In forward bias region, defect assisted tunneling is most likely the dominant mechanism. The existence of defect assisted tunneling is due to the presence of threading dislocation and V-defects that eventually resulted in leakage current. In the range of $0.3 \mathrm{~V} \sim 1.8 \mathrm{~V}$, electron carriers are dominant in low forward bias while in the range of $1.6 \mathrm{~V} \sim 2.5 \mathrm{~V}$, hole carriers are dominant in the medium forward bias region.

4) The region of bias is not the only parameters that can determine the characteristics of the mechanism. By analyzing the dependence of measured data against temperature, it can be said that the VRH is dominant in the reverse bias region whose temperature of conduction is below $200 \mathrm{~K}$. It is important to understand the temperature dependence of leakage current because there is a possibility for the performance of the LED to be affected by temperature in an extremely hot or cold countries [23].

In summary, we have reviewed the dominant mechanism according to the region of bias and temperature dependencies. These address the key questions regarding the characteristics of mechanism that is dominant in reverse and forward leakage current for nitride based LED. Some issues need to be addressed, such as the type of defects which become the cause for the existence of leakage current 
defects which become the cause for the existence of leakage current mechanism and further understanding on ways to reduce the amount of leakage current in LED.

\section{REFERENCES}

[1] Jeon, S.-K., Lee, J.-G., Park, E.-H., Jang, J., Lim, J.-G., Kim, S.-K., \& Park, J.-S. (2009). The effect of the internal capacitance of InGaN-light emitting diode on the electrostatic discharge properties. Applied Physics Letters, 94(13), 131106.

[2] Meneghini, M., Tazzoli, A., Mura, G., Meneghesso, G., \& Zanoni, E. (2010). A review on the physical mechanisms that limit the reliability of GaN-Based LEDs. IEEE Transactions on Electron Devices, 57(1), 108118.

[3] Meneghini, M., Trivellin, N., Butendeich, R., Zehnder, U., Hahn, B., Meneghesso, G., \& Zanoni, E. (2010). Reliability of InGaN-based LEDs submitted to reverse-bias stress. physica status solidi (c), 7(7-8), 22082210 .

[4] Han, D.-P., Oh, C.-H., Zheng, D.-G., Kim, H., Shim, J.-I., Kim, K.-S., \& Shin, D.-S. (2014). Analysis of nonradiative recombination mechanisms and their impacts on the device performance of InGaN/GaN lightemitting diodes. Japanese Journal of Applied Physics, 54(2S), 02BA01.

[5] Jung, E., \& Kim, H. (2014). Analysis of GaN-based light-emitting diodes degraded by generation of deep-level states. physica status solidi (a), 211(8), 1764-1768.

[6] Liu, J., Wong, H., Siu, S. L., Kok, C. W., \& Filip, V. (2012). Degradation behaviors of GaN light-emitting diodes under high-temperature and highcurrent stressing. Microelectronics Reliability, 52(8), 1636-1639.

[7] Cao, X. A., Sandvik, P. M., LeBoeuf, S. F., \& Arthur, S. D. (2003) Defect generation in InGaN/GaN light-emitting diodes under forward and reverse electrical stresses. Microelectronics Reliability, 43(12), $1987-$ 1991.

[8] Yang, S.-C., Lin, P., Wang, C.-P., Huang, S. B., Chen, C.-L., Chiang, P.F., ... Chu, M.-T. (2010). Failure and degradation mechanisms of highpower white light emitting diodes. Microelectronics Reliability, 50(7), 959-964.

[9] Han, D.-P., Oh, C.-H., Kim, H., Shim, J.-I., Kim, K.-S., \& Shin, D.-S. (2015). Conduction mechanisms of leakage currents in InGaN/GaN-Based light-emitting Diodes. IEEE Transactions on Electron Devices, 62(2), 587-592.

[10] Kim, J., Tak, Y., Hong, H.-G., Yang, M., Chae, S., Park, J., ... Chung, U.I. (2012). Investigation of reverse leakage characteristics of InGaN/GaN light-emitting Diodes on silicon. IEEE Electron Device Letters, 33(12), $1741-1743$

[11] Kim, K.-S., Kim, J.-H., \& Cho, S. N. (2011). Leakage current characteristics of Nitride-Based InGaN light-emitting Diode. IEEE Photonics Technology Letters, 23(8), 483-485.

[12] Ganichev, S. D., Ziemann, E., Prettl, W., Yassievich, I. N., Istratov, A. A., \& Weber, E. R. (2000). Distinction between the Poole-Frenkel and tunneling models of electric-field-stimulated carrier emission from deep levels in semiconductors. Physical Review B, 61(15), 10361-10365.

[13] Cao, X. A., Stokes, E. B., Sandvik, P. M., LeBoeuf, S. F., Kretchmer, J. \& Walker, D. (2002). Diffusion and tunneling currents in GaN/InGaN multiple quantum well light-emitting diodes. IEEE Electron Device Letters, 23(9), 535-537.

[14] Shan, Q., Meyaard, D. S., Dai, Q., Cho, J., Fred Schubert, E., Kon Son, J., \& Sone, C. (2011). Transport-mechanism analysis of the reverse leakage current in GaInN light-emitting diodes. Applied Physics Letters, 99(25), 253506.

[15] Miller, E. J., Yu, E. T., Waltereit, P., \& Speck, J. S. (2004). Analysis of reverse-bias leakage current mechanisms in GaN grown by molecularbeam epitaxy. Applied Physics Letters, 84(4), 535.

[16] Kim, J., Tak, Y., Chae, S., \& Park, Y. (2013). Effect of V-Shaped pit size on the reverse leakage current of InGaN/GaN light-emitting Diodes. IEEE Electron Device Letters, 34(11), 1409-1411.

[17] Kim, T.-S., Ahn, B.-J., Dong, Y., Park, K.-N., Lee, J.-G., Moon, Y., ... Song, J.-H. (2012). Well-to-well non-uniformity in InGaN/GaN multiple quantum wells characterized by capacitance-voltage measurement with additional laser illumination. Applied Physics Letters, 100(7), 071910.

[18] Kim, J., Tak, Y., Chae, S., \& Park, Y. (2013). Analysis of forward tunneling current in InGaN/GaN multiple quantum well light-emitting diodes grown on Si (111) substrate. Journal of Applied Physics, 114(1), 013101.

[19] Yan, D., Lu, H., Chen, D., Zhang, R., \& Zheng, Y. (2010). Forward tunneling current in GaN-based blue light-emitting diodes. Applied Physics Letters, 96(8), 083504.

[20] Han, D.-P., Zheng, D.-G., Oh, C.-H., Kim, H., Shim, J.-I., Shin, D.-S., \& Kim, K.-S. (2014). Nonradiative recombination mechanisms in
InGaN/GaN-based light-emitting diodes investigated by temperaturedependent measurements. Applied Physics Letters, 104(15), 151108.

[21] Reynolds, C. L., \& Patel, A. (2008). Tunneling entity in different injection regimes of $\mathrm{InGaN}$ light emitting diodes. Journal of Applied Physics, 103(8), 086102.

[22] Auf der Maur, M., Galler, B., Pietzonka, I., Strassburg, M., Lugauer, H., \& Di Carlo, A. (2014). Trap-assisted tunneling in InGaN/GaN singlequantum-well light-emitting diodes. Applied Physics Letters, 105(13), 133504.

[23] Wooi, C. L., Abdul-Malek, Z., \& Mashak, S. V. (2013). Effect of Ambient temperature on leakage current of Gapless metal oxide surge Arrester. Jurnal Teknologi, 64(4).

[24] Crawford, M. H. (2009). LEDs for solid-state lighting: Performance challenges and recent advances. IEEE Journal of Selected Topics in Quantum Electronics, 15(4), 1028-1040.

[25] Meneghini, M., Vaccari, S., Lago, M. D., Marconi, S., Barbato, M., Trivellin, N., ... Zanoni, E. (2014). ESD degradation and robustness of RGB LEDs and modules: An investigation based on combined electrical and optical measurements. Microelectronics Reliability, 54(6-7), 11431149.

[26] Kozlowski, G., Corbett, B., \& Schulz, S. (2015). Color stability, wave function overlap and leakage currents in InGaN-based LED structures: The role of the substrate orientation. Semiconductor Science and Technology, 30(5), 055014.

[27] Nakamura, S. (2009). Current status of GaN-Based solid-state lighting. MRS Bulletin, 34(02), 101-107.

[28] Jung, E., Lee, J. K., Kim, M. S., \& Kim, H. (2015). Leakage current analysis of GaN-Based light-emitting Diodes using a parasitic Diode model. IEEE Transactions on Electron Devices, 62(10), 3322-3325.

[29] Ferdous, M. S., Wang, X., Fairchild, M. N., \& Hersee, S. D. (2007). Effect of threading defects on InGaNGaN multiple quantum well light emitting diodes. Applied Physics Letters, 91(23), 231107.

[30] Kum, H., Kim, M., Lee, D., Tak, Y., Maeng, J., Kim, J., .. Park, Y. (2015). Dependence of reverse bias leakage on depletion width and V-pit size in InGaN/GaN light-emitting diodes grown on silicon. Journal of Vacuum Science \& Technology B, Nanotechnology and Microelectronics: Materials, Processing, Measurement, and Phenomena, 33(6), 060602.

[31] Kim, G., Park, E., Kim, J. H., Bae, J.-H., Kang, D. H., \& Park, B.-G. (2014). Analysis of trap and its impact on InGaN-based blue lightemitting diodes using current-transient methodology. Japanese Journal of Applied Physics, 53(6), 062101.

[32] Moroz, V., Wong, H. Y., Choi, M., Braga, N., Mickevicius, R. V., Zhang, Y., \& Palacios, T. (2016). The impact of defects on GaN device behavior: Modeling dislocations, traps, and pits. ECS Journal of Solid State Science and Technology, 5(4), P3142-P3148.

[33] Kizilyalli, I. C., Prunty, T., \& Aktas, O. (2015). 4-kV and 2.8-m $\Omega-\mathrm{cm}^{\wedge} 2$ Vertical GaN p-n Diodes With Low Leakage Currents. IEEE Electron Device Letters, 36(10), 1073-1075.

[34] Zhang, Y., Sun, M., Wong, H.-Y., Lin, Y., Srivastava, P., Hatem, C., ... Palacios, T. (2015). Origin and control of oFF-state leakage current in GaN-on-Si vertical Diodes. IEEE Transactions on Electron Devices, 62(7), 2155-2161.

[35] Hsu, J. W. P., Manfra, M. J., Lang, D. V., Richter, S., Chu, S. N. G., Sergent, A. M., ... Molnar, R. J. (2001). Inhomogeneous spatial distribution of reverse bias leakage in GaN Schottky diodes. Applied Physics Letters, 78(12), 1685.

[36] Miller, E. J., Schaadt, D. M., Yu, E. T., Poblenz, C., Elsass, C., \& Speck, J. S. (2002). Reduction of reverse-bias leakage current in Schottky diodes on GaN grown by molecular-beam epitaxy using surface modification with an atomic force microscope. Journal of Applied Physics, 91(12), 9821.

[37] Mandurrino, M., Verzellesi, G., Goano, M., Vallone, M., Bertazzi, F., Ghione, G., ... Zanoni, E. (2015). Physics-based modeling and experimental implications of trap-assisted tunneling in InGaN/GaN lightemitting diodes. physica status solidi (a), 212(5), 947-953.

[38] Cao, X. A., Teetsov, J. M., D’Evelyn, M. P., Merfeld, D. W., \& Yan, C. H. (2004). Electrical characteristics of InGaN/GaN light-emitting diodes grown on GaN and sapphire substrates. Applied Physics Letters, 85(1), 7.

[39] Hirsch, L., \& Barrière, A. S. (2003). Electrical characterization of InGaN/GaN light emitting diodes grown by molecular beam epitaxy. Journal of Applied Physics, 94(8), 5014.

[40] Zhi, T., Tao, T., Liu, B., Li, Y., Zhuang, Z., Zhang, G., ... Zheng, Y. (2015). Asymmetric tunneling model of forward leakage current in GaN/InGaN light emitting diodes. AIP Advances, 5(8), 087151.

[41] Cao, X. A., Sandvik, P. M., LeBoeuf, S. F., \& Arthur, S. D. (2003). Defect generation in InGaN/GaN light-emitting diodes under forward and reverse electrical stresses. Microelectronics Reliability, 43(12), 19871991. 
[42] Mandurrino, M., Goano, M., Vallone, M., Bertazzi, F., Ghione, G., Verzellesi, G., Meneghini, M., Meneghesso, G., Zanoni, E. (2015). Semiclassical simulation of trap-assisted tunneling in GaN-based lightemitting diodes. Journal of Computational Electronics, 14(2), 444-455.

[43] Zhu, D., Xu, J., Noemaun, A. N., Kim, J. K., Schubert, E. F., Crawford, M. H., \& Koleske, D. D. (2009). The origin of the high diode-ideality factors in $\mathrm{GaInN} / \mathrm{GaN}$ multiple quantum well light-emitting diodes. Applied Physics Letters, 94(8), 081113.

[44] Casey, H. C., Muth, J., Krishnankutty, S., \& Zavada, J. M. (1996). Dominance of tunneling current and band filling in InGaN/AlGaN double heterostructure blue light-emitting diodes. Applied Physics Letters, 68(20), 2867.

[45] Perlin, P., Osiński, M., Eliseev, P. G., Smagley, V. A., Mu, J., Banas, M., Sartori, P. (1996). Low-temperature study of current and electroluminescence in InGaN/AlGaN/GaN double-heterostructure blue light-emitting diodes. Applied Physics Letters, 69(12), 1680.

[46] Mayes, K., Yasan, A., McClintock, R., Shiell, D., Darvish, S. R., Kung, P., \& Razeghi, M. (2004). High-power $280 \mathrm{~nm}$ AlGaN light-emitting diodes based on an asymmetric single-quantum well. Applied Physics Letters, 84(7), 1046.
[47] Lee, S. W., Oh, D. C., Goto, H., Ha, J. S., Lee, H. J., Hanada, T., ... Lee, J. S. (2006). Origin of forward leakage current in GaN-based lightemitting devices. Applied Physics Letters, 89(13), 132117.

[48] Choi, I.-G., Han, D.-P., Yun, J., Kim, K. S., Shin, D.-S., \& Shim, J.-I. (2013). Investigation of dominant Nonradiative mechanisms as a function of current in InGaN/GaN light-emitting Diodes. Applied Physics Express, 6(5), 052105.

[49] Sah, C., Noyce, R., \& Shockley, W. (1957). Carrier generation and recombination in P-N Junctions and P-N junction characteristics. Proceedings of the IRE, 45(9), 1228-1243.

[50] Xu, M., Mu, Q., Xiao, L., Zhou, Q., Wang, H., Ji, Z., \& Xu, X. (2016). Temperature dependent current-voltage curves study of GaN-based blue light-emitting diode. Materials Express, 6(2), 205-209. 